\title{
An In Vitro Hemodynamic Loop Model to Investigate the Hemocytocompatibility and Host Cell Activation of Vascular Medical Devices
}

\author{
Max Wacker ${ }^{1}$, Ulf Betke ${ }^{2}$, Katrin Borucki ${ }^{3}$, Jörn Hülsmann ${ }^{1}$, George Awad ${ }^{1}$, Sam Varghese ${ }^{1}$, Maximilian Scherner ${ }^{1}$, Michael \\ Hansen ${ }^{4}$, Jens Wippermann ${ }^{1}$, Priya Veluswamy ${ }^{1}$ \\ ${ }^{1}$ Department of Cardiothoracic Surgery, Otto-von-Guericke-University ${ }^{2}$ Department of Mechanical Engineering, Institute for Materials and Joining \\ Technology, Otto-von-Guericke-University ${ }^{3}$ Institute of Clinical Chemistry and Pathobiochemistry, Otto-von-Guericke-University ${ }^{4}$ Division of Cardiology and \\ Angiology, Department of Internal Medicine, Otto-von-Guericke-University
}

\section{Corresponding Author}

Max Wacker

max.wacker@med.ovgu.de

\section{Citation}

Wacker, M., Betke, U., Borucki, K., Hülsmann, J., Awad, G., Varghese, S., Scherner, M., Hansen, M., Wippermann, J., Veluswamy, P. An In Vitro Hemodynamic Loop Model to Investigate the Hemocytocompatibility and Host Cell Activation of Vascular Medical Devices. J. Vis. Exp. (162), e61570, doi:10.3791/61570 (2020).

\section{Date Published}

August 21, 2020

DOI

$10.3791 / 61570$

URL

jove.com/video/61570

\section{Abstract}

In this study, the hemocompatibility of tubes with an inner diameter of $5 \mathrm{~mm}$ made of polyvinyl chloride (PVC) and coated with different bioactive conjugates was compared to uncoated PVC tubes, latex tubes, and a stent for intravascular application that was placed inside the PVC tubes. Evaluation of hemocompatibility was done using an in vitro hemodynamic loop model that is recommended by the ISO standard 10993-4. The tubes were cut into segments of identical length and closed to form loops avoiding any gap at the splice, then filled with human blood and rotated in a water bath at $37^{\circ} \mathrm{C}$ for 3 hours. Thereafter, the blood inside the tubes was collected for the analysis of whole blood cell count, hemolysis (free plasma hemoglobin), complement system (sC5b-9), coagulation system (fibrinopeptide A), and leukocyte activation (polymorphonuclear elastase, tumor necrosis factor and interleukin-6). Host cell activation was determined for platelet activation, leukocyte integrin status and monocyte platelet aggregates using flow cytometry. The effect of inaccurate loop closure was examined with x-ray microtomography and scanning electron microscopy, that showed thrombus formation at the splice. Latex tubes showed the strongest activation of both plasma and cellular components of the blood, indicating a poor hemocompatibility, followed by the stent group and uncoated PVC tubes. The coated PVC tubes did not show a significant decrease in platelet activation status, but showed an increased in complement and coagulation cascade compared to uncoated PVC tubes. The loop model itself did not lead to the activation of cells or soluble factors, and the hemolysis level was low. Therefore, the presented in vitro hemodynamic loop model avoids excessive activation of blood components by mechanical forces 
and serves as a method to investigate in vitro interactions between donor blood and vascular medical devices.

\section{Introduction}

Hemocompatibility testing of medical devices is a crucial step in the development of new devices such as vascular stents or perfusion tubes for extracorporeal membrane oxygenation. Until today, animal models are considered as standard tools to finalize the procedure for testing the medical devices prior to its implementation in humans. Henceforth, it is necessary to find alternative in vitro models that further aid in minimizing investigations on animals. In this study, we, therefore, have explored a miniature in vitro hemodynamic loop model. The goal of this presented method is to test the in vitro blood compatibility of medical devices in accordance to the ISO 10993-4 standard.

The ISO 10993-4 standard describes standardized sets of clinical parameters to be investigated on blood specimen ${ }^{1}$. Briefly, these are thrombosis (platelet aggregation and count), coagulation (fibrinopeptide A, FPA), hematological analysis (whole blood cell count), hemolysis index (free plasma hemoglobin) and the complement system (terminal complement complex, sC5b9). However, additional markers, such as neutrophil polymorphonuclear elastase (PMN), interleukin 6 (IL-6) and tumor necrosis factor - alpha (TNF) reflecting the activation status of leukocytes can also be accounted for measurements. To determine and to quantify the circulating cell free proteins that are present in blood plasma, sandwich enzymatic linked immunosorbent assay (ELISA) represents a conventional and most reliable $\operatorname{method}^{2,3}$. Likewise, the phenotype and activation status of the host cells (e.g., leukocytes) can be quantified by detecting the cell surface expression of molecules by flow cytometry (FACS) that provides single cell suspension based readouts, where fluorescent labeled specific antibodies bind to the targeted cell surface molecules ${ }^{4}$. Scanning electron microscopy (SEM) is also recommended to determine thrombus formation on the tested material by the ISO 10993-4 standard $^{1}$. This method can be complemented with X-ray microtomography $(\mu \mathrm{CT})$, to perform structural analysis of the thrombus e.g., its thickness, size and localization in a 3D rendered image ${ }^{5}$.

The rationale behind using this in vitro hemodynamic model is to screen for the best performing and compatible medical devices by understanding the basic physiological dynamics of blood components such as platelets, that are involved in the primary hemostasis or leukocytes and their interaction with different types of vascular devices. Such in vitro systems are highly demanded as they reduce the need for animal studies.

The here presented loop model fulfills these demands. This model was first described by A.B. Chandler in 1958 for the production of blood thrombi and is, therefore, also called Chandler Loop model ${ }^{6}$. Until now, this model has been used in a series of experiments and modifications to investigate the blood biocompatibility of medical devices ${ }^{7}, 8,9,10,11,12,13,14$. It consists of polymer tubes, which are partly filled with blood and shaped into re-closable loops. These loops rotate in a temperaturecontrolled water bath to simulate vascular flow conditions with its hemorheological effects. Alternative methods such as pump driven models or models that use mechanical ball valves inside the loops to induce a blood flow inside the 
polymer tubes have already been described ${ }^{15,16}$. However, the overall advantage of the here presented method is that the mechanical force applied to the blood cells and proteins is low, avoiding hemolysis, and there is no contact between blood and connectors, that could possibly lead to flow turbulences and activation of blood components. The main activating factors inside the loop are the test material itself and the air that is trapped inside. This helps to minimize sources of measuring error and to deliver a high reproducibility, even if the blood-air interface can lead to protein denaturation ${ }^{17}$. It is also possible to investigate varieties of tubing materials and stent diameters without length or size restrictions thereby allowing the use of tubes of different length and inner diameter. Moreover, host hemocompatibilities on inaccurate loop closure and exposure to the uncoated tube surface are also possible to investigate. Other similar medical applications of this in vitro hemodynamic loop model is that it could also be used to study the interactions between immunotherapeutics (drugs) and blood components during either preclinical development or individual drug safety screening prior to first-in-man phase I clinical trial, or for the generation of thrombus material that can be used in further experiments ${ }^{18,19,20}$.

This study describes a detailed protocol for testing the hemocompatibilities of perfusion tubes and/or stents. Here, the comparison between uncoated and coated PVC tubes (hepPVC: heparin coating, polyPVC: coating with an bioactive polymer). Lowered activation of platelets, but a higher activation of the coagulation system (FPA) were found for both coated tubes in comparison to the uncoated tubes. The hepPVC tubes used here are modified with covalently bound heparin to make them thromboresistant ${ }^{21}$ and have already been employed in a loop model to optimize and characterize different parameters ${ }^{22}$. The polyPVC tubes used in this study are commercially available tubes used in clinical settings of extracorporeal blood perfusion and are coated with a heparin polymer to reduce their thrombogenicity 23 . Sometimes, in clinical applications even uncoated PVC tubes are used. Therefore, we included latex tubes as a positive control group that showed excessive activation of platelets, coagulation system, and soluble factors like IL-6, TNF and PMN elastase. Thrombus formation was noticed when inaccurate loop closure was simulated. This led to the activation of coagulation and complement system as well as leukocytes and platelets compared to the baseline conditions. Furthermore, blood contact to the here used stent material (bare metal nitinol stent, covered with carbon-impregnated expanded polytetrafluoroethylene) led to higher platelet and leukocyte activation in terms of PMN elastase. Overall, the presented model did not induce hemolysis in any of the tested vascular devices as they were comparable to the baseline or static conditions, except for the latex tubes, where red blood cell (RBC) hemolysis was obvious. Moreover, these perfusion tubes can be examined either by imaging or by histology. Though histological evaluations might be feasible, we mainly focused on ELISA and flow cytometry to perform these experiments and thereby enabling the feasibilities of conducting experiments based on the here presented model for many laboratories. Thus, this method represents a feasible method to test the blood biocompatibility of vascular medical devices in accordance with the recommendations of the ISO 10993-4 standard. Furthermore, this method can be used whenever an interaction between blood and materials should be tested under flow conditions, mimicking the in vivo conditions. 


\section{Protocol}

This study was approved by the Ethics Committee of the medical faculty of the University Hospital Magdeburg (application number 88/18) and the subjects provided written informed consent prior to the blood drawing procedure.

\section{Heparin stock preparation and blood sampling}

1. Calculate the amount of blood needed for the entire experiments.

NOTE: Almost, $5 \mathrm{~mL}$ of heparinized blood is required for each vascular device in duplicates (loops). Likewise, $5 \mathrm{~mL}$ of blood is required for each baseline and static condition which is kept aside at room temperature.

2. Prepare a heparin stock solution at the concentration of $100 \mathrm{IU} / \mathrm{mL}$ by diluting the unfractionated heparin (e.g., Rotexmedia) in deionized water. Use $150 \mu \mathrm{L}$ of this solution for the heparinization of $10 \mathrm{~mL}$ fresh blood.

3. Calculate the amount of blood as well as plasma that are needed for whole blood cell count, ELISA and FACS assays.

NOTE: The measurements represented in this study are obtained from two loops running in parallel (one as duplicate) with a total blood volume of $10 \mathrm{~mL}$.

4. Based on the required amount of blood, fill $10 \mathrm{~mL}$ syringes with $150 \mu \mathrm{L}$ of heparin stock solution to prevent blood coagulation with a final heparin concentration of $1.5 \mathrm{IU} /$ $\mathrm{mL}$ blood.

5. Draw blood using a butterfly (size: $21 \mathrm{G}$ ). Fill the syringes very gently to avoid hemolysis or cell activation due to excessive vacuum.

NOTE: Permission from the local ethical committee should be obtained before the commencement of the experiments. Obtain informed consent from each human blood donor. Ensure that the blood donor is healthy and does not take any medication, especially no antiplatelet agents or non-steroidal anti-inflammatory drugs for at least 10 days prior to the experiments. Of note, same donor is preferred when comparing different types of vascular devices for the first time as presented in this manuscript. To further evaluate the inter-individual differences, the protocol can be repeated with different donors.

6. Collect blood in a glass beaker, avoid excessive agitation.

\section{In vitro hemodynamic loop assembly}

1. Fill the water bath until the water level reaches up to the center of the rotation unit. Set water temperature to $37^{\circ} \mathrm{C}$.

2. Loop assembly for testing different tubing materials (polyPVC, hepPVC, PVC and latex)

1. Cut two $50 \mathrm{~cm}$ long pieces of each tube material (inner diameter $5 \mathrm{~mm}$ ) with the tube cutter. Ensure that the cutting surface is flat, as this is particularly important for perfect closure for the loops with small diameters so that blood flows without any distortion on the fitting edge.

NOTE: This entire protocol utilizes the tubes with an inner diameter of $5 \mathrm{~mm}$.

2. To ease the handling and to avoid post-sample processing delay after rotation, run only four loops ( 2 materials in duplicates) in parallel.

3. To generate a loop shape, plug the open endings of the tubes into a short piece of silicon tube fitting the outer diameter of the investigative tube.

4. Use the polycarbonate tension bands to ensure proper closing of the loops. When used for the first 
time, adjust the length of the bands to fit the outer diameter of the loop by cutting the tension band with scissors in required lengths and secure it with a $3 \mathrm{~mm}$ torque wrench.

5. Carefully tighten the locking screw of the tension band connector under inspection of the tube endings. Adjust the closing force so that no gap remains between the tube endings. If the locking screw is totally tightened and the tension of the polycarbonate band seems too low to close the gap between the tube endings, open the locking the system and cut a few $\mathrm{mm}$ of the tension band. Repeat this, until accurate loop closure is achieved (Figure 1A).

6. If the tubing material is very soft and tends to slip inside the tension bands, fix the loop with electrical tape to the tension band (Figure 1B,C).

7. Prepare an additional loop of PVC for temperature control with the same dimensions as the test loops.

8. Secure the loops in the loop cradle of the rotation unit outside the water bath. Thereafter, attach the loop cradle to the rotation unit inside the water bath (Figure 1E).

9. Dismantle the tension band partly from the loops and unplug one end of each tube to open the loop.

10. Gently fill each loop with $5 \mathrm{~mL}$ of blood with a $5 \mathrm{~mL}$ serological pipette. Mix blood gently twice in the glass beaker by slowly pipetting up and down before loading into the loops.

11. Take the disposable thermometer and place it inside the temperature control loop. If the thermometer is too large, cut it with scissors to fit smaller tubes. Fill the loop with $5 \mathrm{~mL}$ of deionized water at room temperature.
12. Close the loops and ensure whether the loops, the tension bands and the rack are properly fitted.

13. Set rotation speed to 30 rounds per minute (rpm) and rotate for $3 \mathrm{~h}$.

3. Loop assembly for testing the impact of improper loop closure (gap)

1. Prepare four loops (polyPVC) as described in 2.2.

2. Close two of the loops properly with the tension bands and avoid any gap between the tube endings.

3. For the other two loops plug the open endings into the bigger tube as described in 2.2.3, but leave a gap of 1-2 $\mathrm{mm}$ in between the loop endings. Do not use the tension bands for these loops (Figure 1D).

4. Prepare one temperature control loop as described in 2.2.7 and 2.2.11.

5. Fill all loops with blood as described in 2.2.10.

6. Set the rotation speed to $30 \mathrm{rpm}$ and rotate for $3 \mathrm{~h}$.

4. Loop assembly for stent testing

1. Prepare four loops as described in 2.2 and following. NOTE: To assess the blood biocompatibility of stents, the tubing material itself should be tested to be biocompatible in order to prevent masking of cell activation. If no data exists, the tube material itself can be tested as described in 2.2. Furthermore, the diameter range within the stent can be applied (see manufacturer's instructions) and should fit the inner diameter of the tube material.

2. Open two of the loops and take the tube out of the tension band system.

3. Insert the stent into the middle of the tube as per the manufacturer's instructions. 
4. Use the other two loops without stent as control. Fill all loops with blood as described in 2.2.10.

5. Set rotation speed to $30 \mathrm{rpm}$ and rotate for $3 \mathrm{~h}$.

5. Temperature control

1. At any time during duration and when rotation is stopped, the temperature of the blood inside the loops is indicated by the thermometer inside the temperature control loop. To read off the temperature, stop the rotation and immediately read off the temperature indicated by the thermometer.

\section{Blood sample processing}

1. After rotation, let the loops stand in the rack for $2 \mathrm{~min}$ in an upward position to let the blood accumulate at the bottom of the loops, avoiding any spilling while opening the loops.

2. Inspect the blood left for static conditions: If this blood is coagulated, an improper heparinization is ultimately suspected. In this case, repeat the experiment preferably also with another blood donor.

3. Carefully take the loops out of the rack. Open the connectors and let the blood flow into a $10 \mathrm{~mL}$ glass beaker.

4. Pool the blood from the tubes that are run in duplicates.

5. Draw fresh blood for baseline analysis from the same donor as described in 1.5 and 1.6.

6. Collection of sodium citrate blood

1. For the collection of sodium citrate blood, fill four $\mathbf{1 . 5}$ $\mathrm{mL}$ tubes, each with $111 \mu \mathrm{L}$ sodium citrate solution collected from sodium citrate tubes, to generate a final concentration of $3.2 \%$ of sodium citrate.

2. Fill each tube with $1 \mathrm{~mL}$ of blood from the glass beakers as described in 1.6 and 3.3.
3. Keep the blood at room temperature and use the this blood for FACS analyses as described in 12.

NOTE: To change the length of the loops for different experimental setups, properly plan aliquots based on the amount of measurements to be made. It may be necessary to establish all required measurements with fresh blood prior to the real experiments to ensure the volume of blood in the loops is enough for all measurements.

7. Collection of ethylenediaminetetraacetic acid (EDTA) blood and plasma samples.

1. From each glass beaker with blood (see 1.6 and 3.3) transfer $4.5 \mathrm{ml}$ of blood into one $5 \mathrm{ml}$ EDTA tube. Fill two EDTA tubes from each $10 \mathrm{ml}$ glass beaker with blood.

2. Gently mix the blood and keep it on ice.

3. Transfer $2 \mathrm{~mL}$ of blood into a $2 \mathrm{~mL}$ locking centrifugation tube and use this blood for blood cell count and free hemoglobin measurement as described in 5 . And 6.

4. Centrifuge the remaining blood at 3,500 $x \mathrm{~g}$ for $20 \mathrm{~min}$.

5. Carefully collect the plasma in the supernatant in 500 $\mu \mathrm{L}$ aliquots and immediately freeze at $-80^{\circ} \mathrm{C}$.

\section{Scanning electron microscopy and $\mu \mathrm{CT}$ images}

1. After blood sample processing, rinse the emptied loops prepared as described in 2.3 with $10 \mathrm{~mL}$ of phosphatebuffered saline (PBS) each.

2. Carefully cut off a $1 \mathrm{~cm}$ long sample from each end of each tube with a scalpel.

3. Incubate sample overnight at $4{ }^{\circ} \mathrm{C}$ in a $2 \%$ glutaraldehyde solution. 
CAUTION: Inhalation of aldehyde vapors can cause nasal symptoms such as a runny nose ore persistent stuffiness and airway irritation, and contact with skin causes dermatitis. Aldehydes should be handled in a fume-hood while wearing gloves, a protective gown and safety goggles.

4. Rinse the sample (3 times) with PBS.

5. Prepare a $1 \%$ solution of osmium tetroxide $\left(\mathrm{OsO}_{4}\right)$ in deionized water and incubate sample for $15 \mathrm{~min}$ at room temperature.

CAUTION: $\mathrm{OsO}_{4}$ is a strong oxidizing agent, it can be reduced by exposure to light. To avoid the reduction during preparation, store $\mathrm{OsO}_{4}$ in a brown glass bottle. $\mathrm{OsO}_{4}$ is extremely volatile, its fumes are toxic to eyes, nose and throat. Always work under a fume-hood and use gloves and protecting clothing, ensuring that no part of the body is exposed to $\mathrm{OsO}_{4}$. Contact your institution's guidelines regarding handling of waste disposal and storage. In general, $\mathrm{OsO}_{4}$ is storable for several months, but it needs special glass bottles with Teflon liner and a desiccator, because $\mathrm{OsO}_{4}$ can discolor internal surfaces and contents of the refrigerator in the presence of leaking fumes.

6. Take out samples, transfer them in a new $15 \mathrm{~mL}$ centrifuge tubes and rinse samples 3 times with PBS.

7. Prepare a series of ethanol with varying concentrations $(25 \%, 50 \%, 75 \%, 95 \%, 100 \%)$

8. Dehydrate samples in ethanol: incubate for 20 min each in $25 \%, 50 \%, 75 \%$ and $90 \%$ and 30 min in $100 \%$.

9. Take samples out of $100 \%$ ethanol and let it air dry overnight at room temperature.
10. Examine samples in the scanning electron microscope at an acceleration voltage of $5 \mathrm{kV}$ and with the X-ray $\mu \mathrm{CT}$ scanner.

\section{Blood cell count}

1. Take $2 \mathrm{~mL}$ of the EDTA blood obtained as described in 3.7 .3

2. Insert the tube into the automated hematology analyzer and follow the manufacturer's instructions.

\section{Measurement of free hemoglobin $(\mathrm{fHb})$ in plasma}

1. Thaw one plasma sample from each condition obtained as described in 3.7.5. Store on ice after thawing.

NOTE: Always thaw frozen plasma samples in a water bath at $37^{\circ} \mathrm{C}$ and immediately transfer to ice, containing some water, to lower the temperature. This is important to avoid activation of blood components during thawing.

2. Use the $\mathrm{fHb}$ reagent and follow the manufacturer's instructions. Avoid contamination after opening and protect the reagent from direct light (sun, UV light).

3. Use a pipetting scheme (see manufacturer's instructions) with 1:5 dilution.

4. Add $1000 \mu \mathrm{L}$ of the hemoglobin reagent in a $1.6 \mathrm{~mL}$ semimicro cuvette. Use this cuvette to determine the blank value.

5. Add $1000 \mu \mathrm{L}$ of the hemoglobin reagent and $250 \mu \mathrm{L}$ of the plasma sample in another semi-micro cuvette.

6. Mix the contents in both cuvettes by flushing the pipette thoroughly by repeatedly filling with reaction mixture, and incubate at least 3 min at room temperature. 
7. Determine the extinction (E) of the sample against $\mathrm{fHb}$ reagent as blank reagent. Calculate the $\mathrm{fHb}$ concentration $(\mathrm{mmol} / \mathrm{l})$ of the sample: $\mathrm{E}_{540}-\mathrm{E}_{680} \times 0.452$.

\section{Measurement of FPA}

1. Thaw a plasma sample from each condition obtained as described in 3.7.5 and store on ice after thawing.

2. Use the FPA Elisa kit and follow the manufacturer's instructions.

\section{Measurement of sC5b9}

1. Thaw a plasma sample from each condition obtained as described in 3.7.5 and store on ice after thawing.

2. Use the sC5b-9 ELISA kit and follow the manufacturer's instructions.

\section{Measurement of PMN}

1. Thaw a plasma sample from each condition obtained as described in 3.7.5 and store on ice after thawing.

2. Use the PMN-Elastase ELISA kit and follow the manufacturer's instructions.

\section{Measurement of TNF}

1. Microplates must be coated one day before running the ELISA. For coating, add $100 \mu \mathrm{L}$ of capture antibody solution to all wells, seal plate and incubate overnight at $2{ }^{\circ} \mathrm{C}-8{ }^{\circ} \mathrm{C}$

2. Thaw one plasma sample from each condition obtained as described in 3.7.5. Store on ice after thawing.

3. Use the TNF ELISA kit and follow the manufacturer's instructions.

\section{Measurement of IL-6}

1. Microplates must be coated with capture antibodies one day prior to the experiment. For coating, add $100 \mu \mathrm{L}$ of capture antibody solution to all wells of the microplate provided with the set, seal plate and incubate overnight at $4{ }^{\circ} \mathrm{C}$

2. Thaw a plasma sample from each condition obtained as described in 3.7.5. and store on ice after thawing.

3. Use the IL-6 ELISA kit and follow the manufacturer's instructions.

\section{FACS analyses}

1. Prepare $500 \mathrm{~mL}$ of FACS buffer by adding $10 \mathrm{~mL}$ of fetal calf serum and $2 \mathrm{~mL}$ of EDTA solution $(0.5 \mathrm{M})$ to $488 \mathrm{~mL}$ of 1x PBS. The FACS buffer can be stored for 4 weeks at $4^{\circ} \mathrm{C}$

2. Use $100 \mu \mathrm{L}$ of the sodium citrate blood (see 3.6.3) for each staining procedure (monocyte platelet aggregates (MPA), platelet activation (PA), leukocyte integrins (LI)).

3. Pipette $100 \mu \mathrm{L}$ of blood from each sample into a $5 \mathrm{~mL}$ FACS tube. From each sample, prepare 3 tubes for antibody staining and label with MPA (monocyte platelet aggregates) panel, PA (platelet aggregates) panel and LI (leukocyte integrin) panel.

4. Mix the rest of the 4 samples into one $5 \mathrm{~mL}$ FACS tube. Use this mix for unstained and fluorescence minus one (FMO) controls.

5. Prepare 6 FACS tubes by pipetting $100 \mu \mathrm{L}$ of the mixed sample blood into each tube. Label 3 tubes as unstained and 3 tubes as FMO-CD41, FMO-CD62P and FMOCD162. 
6. Add $100 \mu \mathrm{L}$ of $4 \%$ paraformaldehyde solution and incubate for $15 \mathrm{~min}$ in the dark at room temperature.

CAUTION: Paraformaldehyde is toxic to skin and eyes. It can cause serious pulmonary irritation when inhaled and can lead to lung damage after prolonged exposure. Furthermore, it is classified as a carcinogen and a reproductive toxin. Always wear gloves and safety glasses and work under the fume hood.

7. Add $1 \mathrm{~mL}$ of wash buffer to each tube and centrifuge at $287 \times g$ for $5 \mathrm{~min}$. Discard the supernatant and repeat wash step two times.

8. For red blood cell (RBC) lysis, add $1 \mathrm{~mL}$ of $1 \mathrm{x}$ buffer RBC lysis buffer (dilute 10x RBC lysis buffer in deionized water), mix by slowly pipetting up and down and incubate for 5 min at RT in the dark.

9. Prepare compensation beads for single stains. To $1 \mathrm{~mL}$ of FACS buffer add 4 drops of each positive and negative beads. Vortex thorough and add $100 \mu \mathrm{L}$ of beads solution to one $5 \mathrm{~mL}$ FACS tube.

10. Prepare 4 tubes with beads and label as CD14-FITC, CD41-BV421, CD45-APC and CD62P-PE. Add $1 \mathrm{~mL}$ of FACS buffer to each tube and centrifuge at $287 \times \mathrm{g}$ for 5 min. Discard supernatant and keep on ice.

11. After RBC lysis, add $1 \mathrm{~mL}$ of FACS buffer to each tube and wash as described in 12.7. Discard supernatant.

12. Prepare antibody cocktails:

1. MPA panel: Add $4 \mu \mathrm{L}$ of CD45-APC, $4 \mu \mathrm{L}$ of CD14FITC and $4 \mu \mathrm{L}$ of CD41-BV421 to $388 \mu \mathrm{L}$ of FACS buffer.

2. PA panel: Add $1.6 \mu \mathrm{L}$ of CD41-BV421 and $12 \mu \mathrm{L}$ of CD62P-PE to $386.4 \mu \mathrm{L}$ of FACS buffer.
3. LI panel: Add $4 \mu \mathrm{L}$ of CD45-APC and $8 \mu \mathrm{L}$ of CD162BV421 to $388 \mu \mathrm{L}$ of FACS buffer. Keep on ice.

13. Prepare single stains: Add $0.5 \mu \mathrm{L}$ to $499.5 \mu \mathrm{L}$ of FACS buffer each for CD14-FITC, CD41-BV421 and CD45APC. For CD62P-PE add $0.3 \mu \mathrm{L}$ to $499.7 \mu \mathrm{L}$ of FACS buffer. Keep on ice.

14. Prepare FMO controls: (i) MPA panel (FMO-CD41): Add 1 $\mu \mathrm{L}$ CD14-FITC antibody and $1 \mu \mathrm{L}$ of CD45-APC antibody to $98 \mu \mathrm{L}$ FACS buffer. (ii) PA panel (FMO-CD62P): Add $0.4 \mu \mathrm{L}$ of CD41-BV421 to $99.6 \mu \mathrm{L}$ of FACS buffer. (iii) $\mathrm{LI}$ panel (FMO-CD162): Add $1 \mu \mathrm{L}$ of CD45-APC to $99 \mu \mathrm{L}$ of FACS buffer. Keep FMO controls on ice.

15. Vortex antibody cocktails and add $100 \mu \mathrm{L}$ of each antibody cocktail as prepared in 12.12 to each of the respective labeled tubes (MPA, PA and $\mathrm{LI}$ panel) as described in 12.3 and mix gently by pipetting up and down. Keep on RT in the dark.

16. Vortex single stain antibody dilutions and add $100 \mu \mathrm{L}$ of the single stain antibody dilutions as prepared in 12.13 . to each of the respective labeled tubes with beads as described in 12.7 and mix gently by pipetting up and down. Keep on RT in the dark.

17. Vortex FMO antibody cocktails and add $100 \mu \mathrm{L}$ of each FMO-1 antibody cocktail as prepared in 12.14. to each of the respective labeled tubes (FMO controls) as described in 12.5. and mix gently by pipetting up and down. Keep at RT in the dark.

18. Incubate all tubes with antibody staining for $30 \mathrm{~min}$ at RT in the dark.

19. Take the three tubes for unstained control (see 12.4) and resuspend pellet in $250 \mu \mathrm{L}$ of FACS buffer. Keep on ice. 
20. After incubation time, wash all tubes except unstained controls one time with FACS buffer as described in 12.7 . Resuspend pellet in in $250 \mu \mathrm{L}$ of FACS buffer and acquire data on the flow cytometer.

21. Use unstained cells for negative settings and compensation mouse beads for positive settings in FACS software. Further, use FMO to control the gating strategy, where FMO-CD41 is used in MPA panel, FMO-CD62P is used in PA panel and FMO-CD162 is used in LI panel.

22. Acquire almost $0.5 \times 10^{6}-0.25 \times 10^{6}$ events per tube for FACS. Save data and analyze with an analysis software (version 9.9.6).

\section{Representative Results}

All presented data, except FACS plots, were analyzed with a statistics software. The FACS plots were analyzed using flow cytometry software.

The analysis of whole blood cell count did not show any significant differences with respect to erythrocytes between all tested conditions (Figure 2). But, platelets and leukocytes were drastically reduced in the latex group, indicating a very poor biocompatibility of latex. This is further underlined by increased levels of free hemoglobin in the latex group, indicating the fact that except for the latex group, none of the other vascular devices or conditions led to extensive hemolysis (Figure 2). Further, the coated PVC tubes, polyPVC and hepPVC, as well as the tested stent did not lead to thrombosis by means of platelet and leukocyte loss, while latex exhibited the highest platelet and leukocyte loss, followed by uncoated PVC tubes that showed a decreased trend.

While all the tested vascular devices led to increased activation of the coagulation system (FPA) and complement component (sC5b-9), the hepPVC loops exhibited a trend for decreased levels of FPA and sC5b-9 when compared specifically to polyPVC loops (Figure 3). Interestingly, uncoated PVC and Gap loops showed lower levels of FPA compared to polyPVC, though not reaching the level of statistical significance. Nevertheless, latex loops exhibited significantly increased levels of FPA when compared to baseline and static conditions.

In accordance with the whole blood cell counts, latex loops exhibited highest levels of TNF, IL-6 and PMN elastase (Figure 4), reaching the level of statistical significance when compared to rest of the groups in terms of TNF and IL-6 (Figure 4A,B), whereas to static and baseline conditions in terms of PMN elastase (Figure 4C). These results indicate the potent activation of leukocytes by latex. The baseline levels of activation markers were always comparable to static conditions, indicating a proper heparinization of the blood.

Interestingly, it was shown that platelet and leukocyte counts for gap induced loops were only slightly reduced with moderate activation of the coagulatory system (FPA) and leukocytes (PMN elastase), though improper loop closure with resulting flow turbulences and blood contact to the uncoated, rough cutting surface led to macroscopically visible clots at the splice (Figure 1F). The clots and its distribution over the whole splice surface were evident with $\mu C T$ and SEM images, while no clot was found when the loops were closed with the external closing device leaving no gap between the loop endings (Figure 5).

Flow cytometric analysis of host blood cells that were stained with platelet specific markers, CD41 and platelet activation marker CD62P, are shown in Figure 6A,B. Here, the latex tubes exhibited exceedingly high median fluorescence intensity (MFI) for CD62P on blood platelets, followed by 
stent, whereas heparin coated polyPVC tubes exhibited minimal activation of platelets depicting anti-thrombogenic property of polyPVC tubes. Furthermore, leukocytes were classified based on the CD45 and SSC (side scatter) based granularity into (i) granulocytes; (ii) monocytes and (iii) lymphocytes (Figure 7), and the expression of $\mathrm{CD}_{162}{ }^{+}$ integrin was detected on each subpopulation of leukocytes that are known to interact with the CD62P on platelets $^{24}$. It was noticed that the integrin expressions were drastically reduced on granulocytes and lymphocytes in latex loops. This result was in line with lowered levels of total frequencies of leukocytes in the latex loops (Figure 2). In general, the integrin levels were higher among monocytes when compared to granulocytes and lymphocytes, indicating the likelihood for the monocyte interaction with activated platelets. In this regard, monocyte platelet aggregates were also evaluated by staining the blood cells with CD14 (as monocyte marker) and CD41 (as platelet marker) and ultimately to identify double positive cells i.e. CD $14^{+} \mathrm{CD} 41^{+}$MPA (Figure 8). Here, we noticed that the stent group exhibited the highest levels of CD41 expression on the MPA, followed by the latex group, indicating an increased tendency to form MPA, despite the reduced frequency of monocyte $(<1 \%)$ in the latex loops.

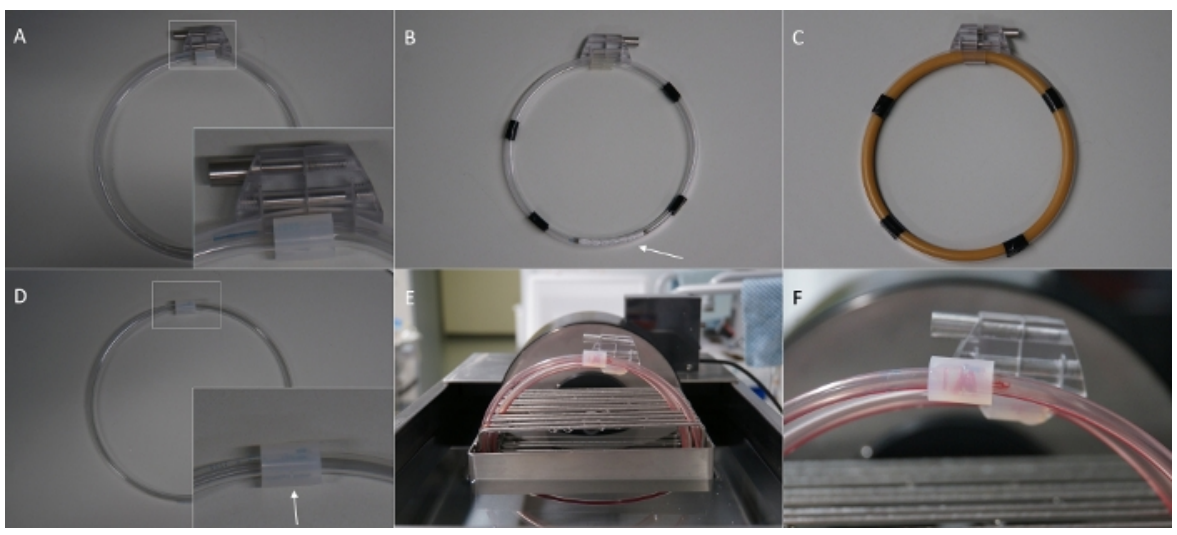

Figure 1: Overview of the in vitro hemodynamic loop model and its modifications. (A) Loop for the gap experiment with external loop closing system, leaving no gap at the splice. (B) Loop made of polyPVC coated PVC tube and stent inside (arrow). (C) Loop made of latex tube. (D) Loop for the gap experiment without the external loop closing system leaving a gap between the tube endings (arrow). (E) Loops placed in the loop cradle inside the water bath and filled with blood. (F) Thrombus resulting in a gap at the splice (arrow) after rotation. Please click here to view a larger version of this figure. 

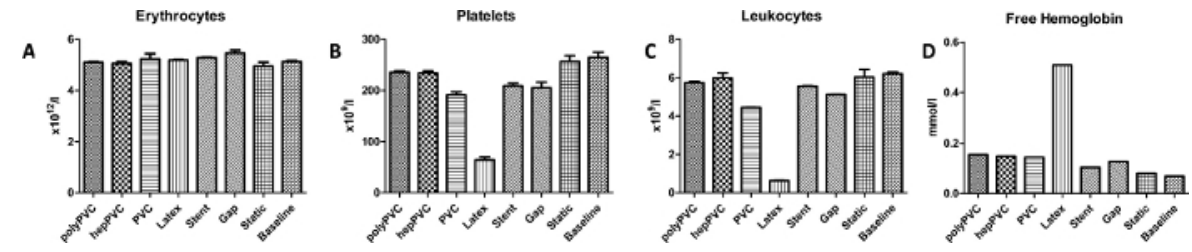

Figure 2: Results for blood cell count and plasma hemoglobin. (A) Erythrocytes count. (B) Platelets count. (F) Leukocytes count. (D) Free plasma hemoglobin. The results indicate the poor biocompatibility of latex, leading to excessive hemolysis. Data are presented as mean value; error bars indicate SEM. $\mathrm{n}=1$. Please click here to view a larger version of this figure.

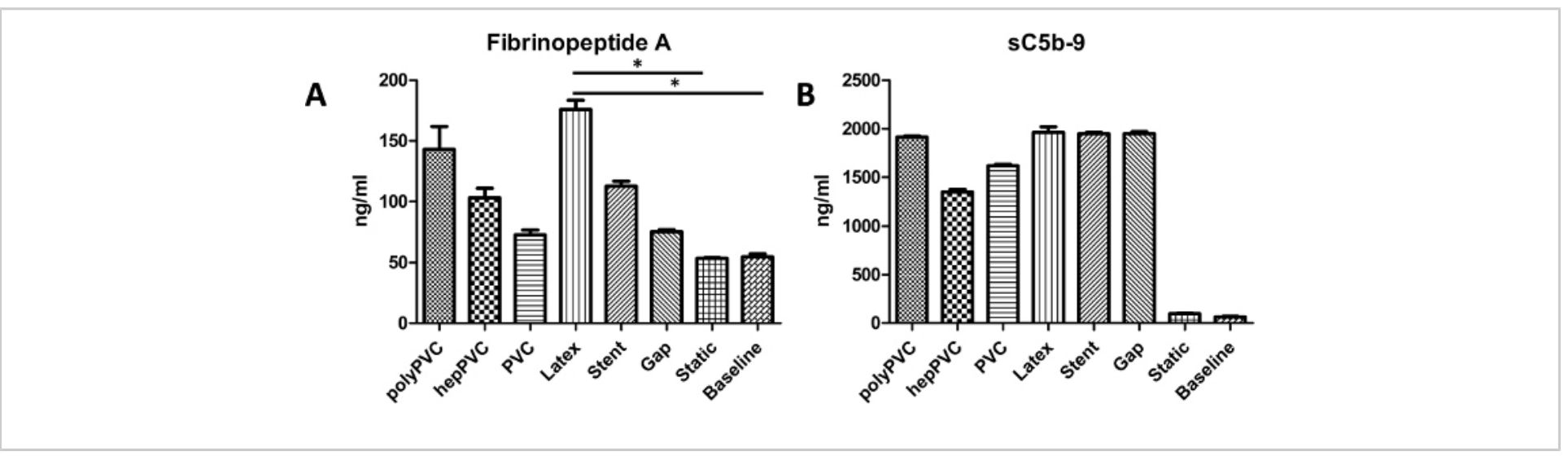

Figure 3: Results for activation of the coagulation and complement system. (A) Coagulation system activation, measured by levels of Fibrinopeptide A (FPA) (B) Complement system activation, measured by levels of sC5b-9. While latex tubes evoked significant elevated levels of the FPA, the complement activation was strong for all tested materials. Data are presented as mean value, error bars indicate SEM. ${ }^{*} p<0.05, n=1$. Please click here to view a larger version of this figure. 

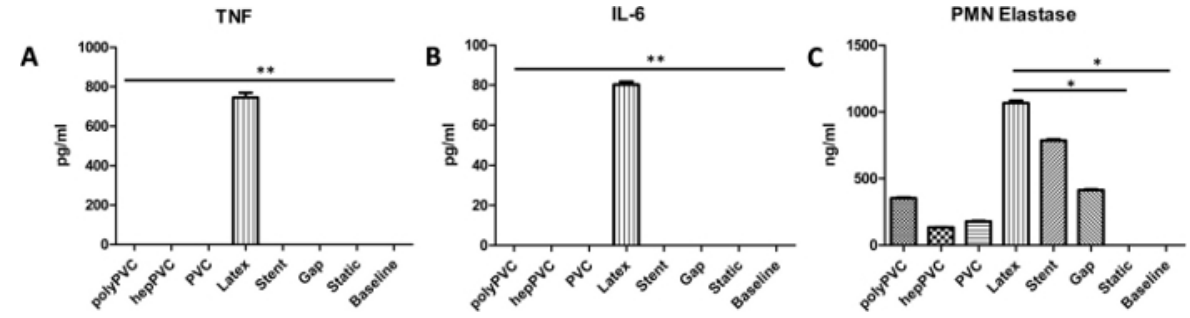

Figure 4: Leukocyte activation markers. (A) Tumor necrosis factor alpha (TNF). (B) Interleukin 6 (IL-6) (C) PMN Elastase. The results indicate increased activation of leukocytes due to elevated levels of the analyzed markers, followed by stent loops, that only led to increased levels for PMN Elastase but not TNF or IL-6. Data are presented as mean value, error bars indicate SEM. ${ }^{*} p<0.5 ;{ }^{* *} p<0.01, n=1$. Please click here to view a larger version of this figure.
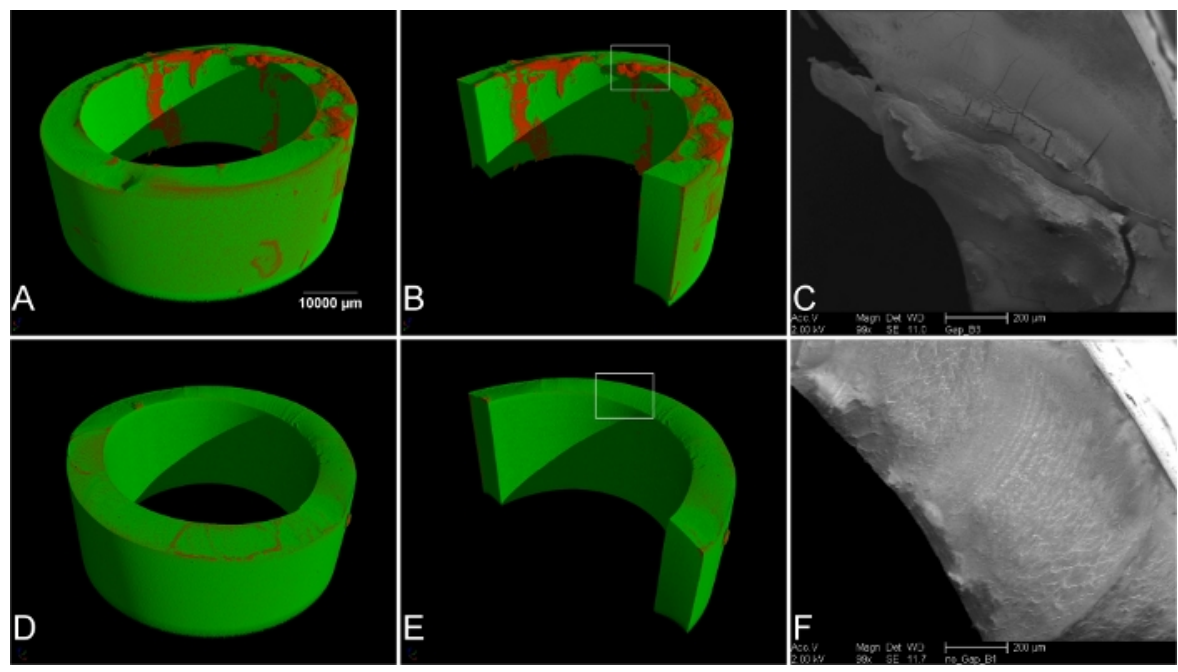

Figure 5: Imaging of the splice of the loops. (A) $\mu$-computer tomography $(\mu \mathrm{CT})$ of loops with improper closing (gap). The red areas indicate thrombus material. (B) Rendering of the luminal side of the tube. The rectangular selection indicates the area for scanning electron microscopy (SEM) (C). (D) $\mu$ CT of loops with external loop closing device and no gap at the splice, and (E) rendering and view of the luminal surface. No thrombus material was found. (F) SEM image of the rectangular selection in (E). No thrombus material was found on the cutting surface. Please click here to view a larger version of this figure. 


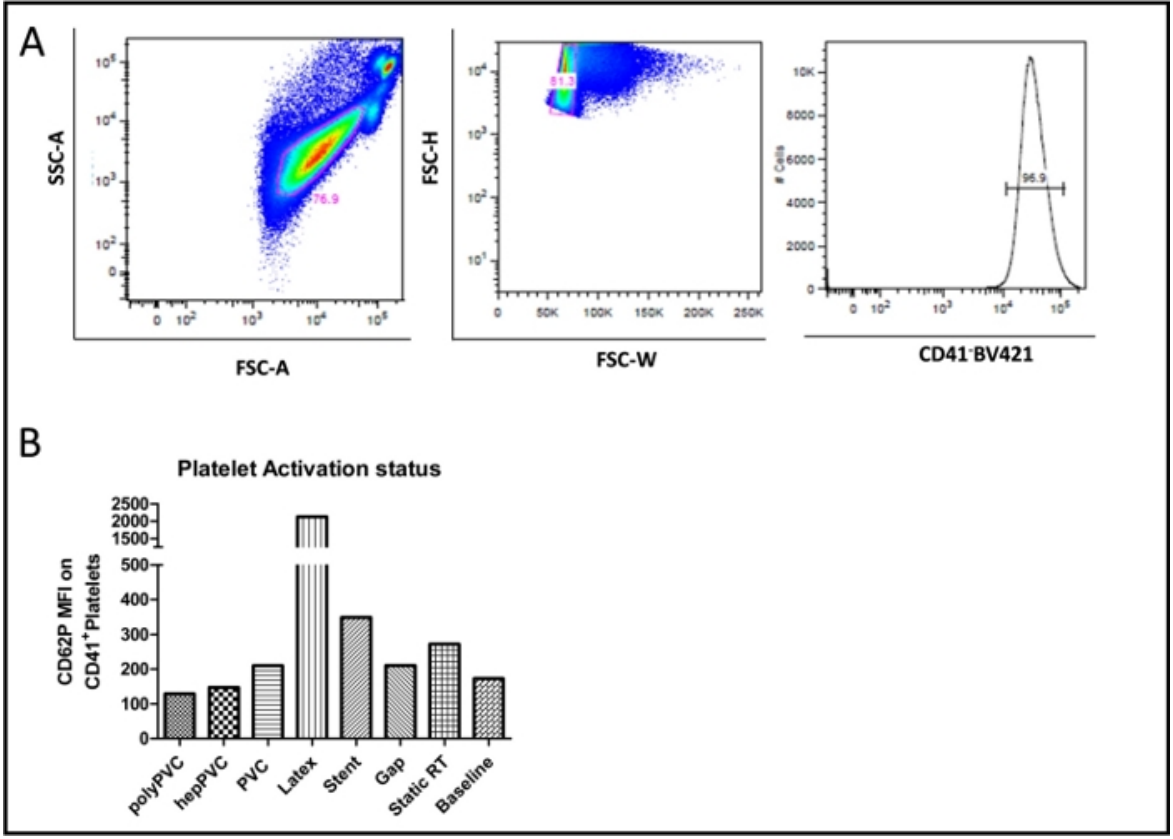

Figure 6: FACS plot for platelet activation (CD62P). (A) Representative FACS plot (basic condition) showing the blood CD41 ${ }^{+}$platelets. (B) Graph showing the platelet activation status reflected by the mean fluorescence intensity (MFI) of the different types of vascular devices in comparison to the static RT and baseline conditions. The data bars present data from single measurements. Please click here to view a larger version of this figure. 


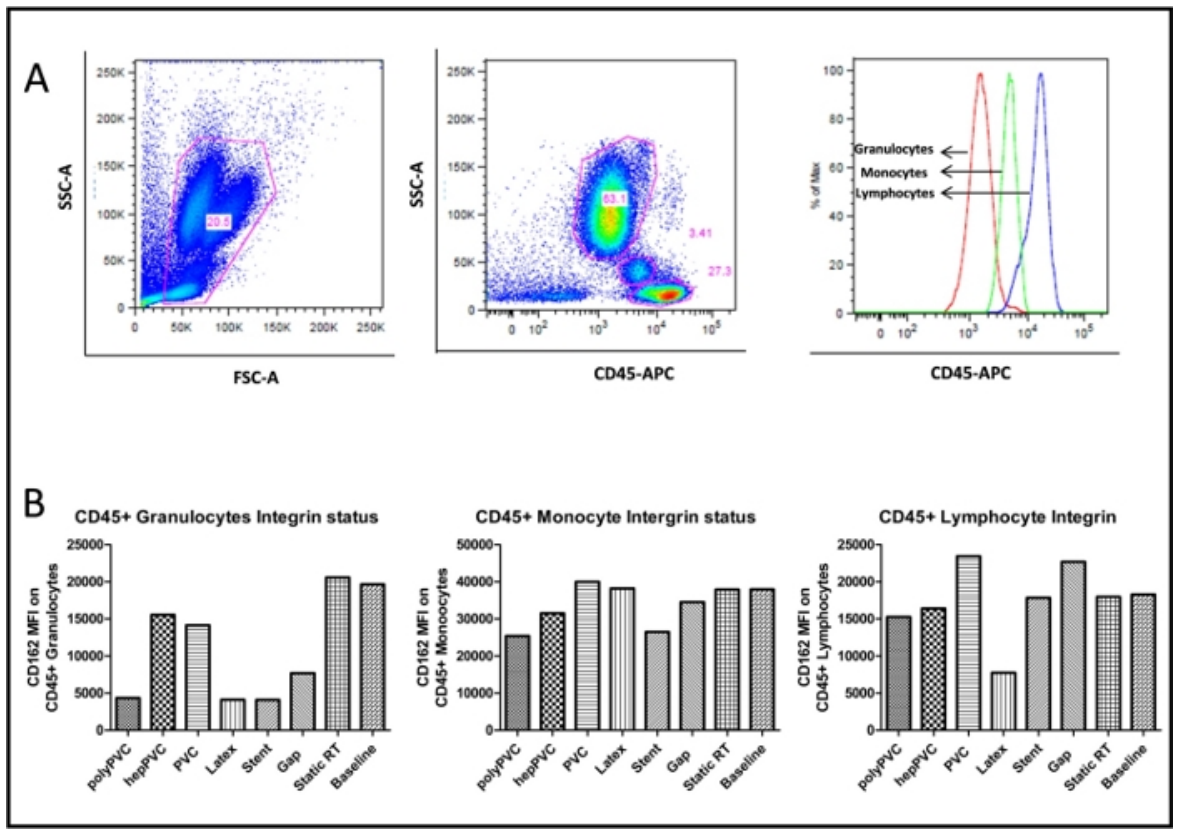

Figure 7: FACS plot for leukocyte integrin (CD162). (A) Representative FACS plot (basic condition) showing the blood $\mathrm{CD}^{4} 5^{+}$leukocytes and subgroups (B) Graph showing the leukocyte CD162 ${ }^{+}$integrin mean fluorescence intensity (MFI) of the different types of vascular devices in comparison to the static and baseline conditions. The data bars present data from single measurements. Please click here to view a larger version of this figure. 


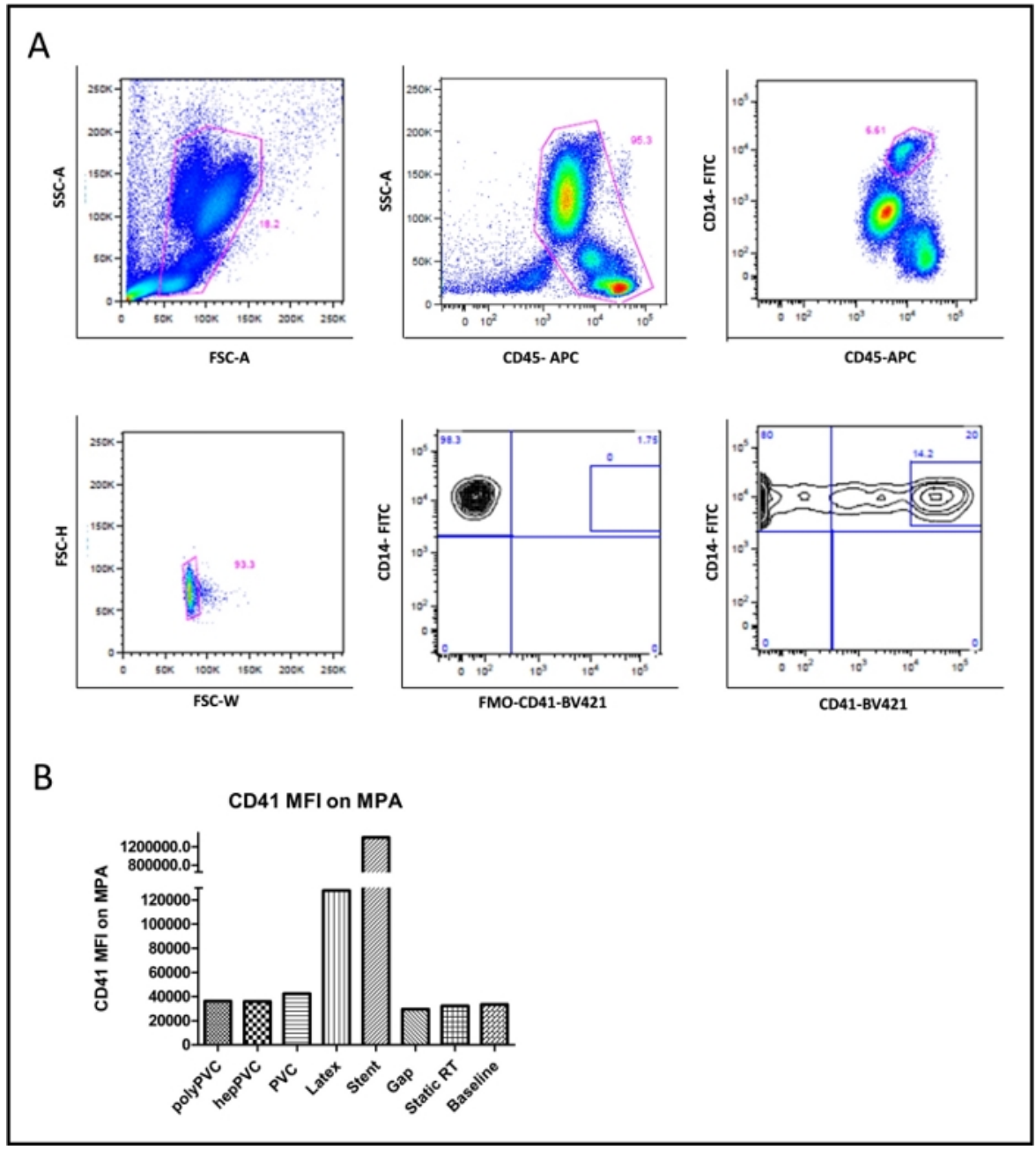

Figure 8: FACS plot for platelet monocyte aggregates (CD41/CD14). (A) Representative FACS plot (basic condition) showing the gating for blood monocytes $\left(\mathrm{CD} 45^{+} / \mathrm{CD} 14^{+}\right)$, platelets $\left(\mathrm{CD} 41^{+}\right)$and monocyte platelet aggregates $\left(\mathrm{CD} 41^{+} /\right.$ $\mathrm{CD}_{14}^{+}$) (B) Graph showing the CD41+ mean fluorescence intensity (MFI) on monocyte platelet aggregates for the various vascular devices compared to the static and baseline conditions. The data bars present data from single measurements. Please click here to view a larger version of this figure.

\section{Discussion}

This study has shown that the presented in vitro hemodynamic loop model offers a reliable method for testing the in vitro blood compatibility of medical devices in accordance to the ISO 10993-4 standard.
Critical steps in the protocol include the drawing of blood and filling the tubes with blood, where excessive vacuum or agitation should be avoided to prevent the blood components from activation by the handling procedure. Furthermore, it is very important to immediately freeze the plasma samples and keep them on ice after thawing, as the complement and 
coagulation system activation can be tampered by keeping the samples on room temperature for a longer time.

Since this model has both merits and demerits when compared to other in vitro models, several factors have to be taken into account while designing the experiments.

First, the loops can be varied in length and diameter to fit various experimental setups. In case the setup includes contrasting tubes of varying inner diameters, it should be kept in mind that the differences in diameter will result in different shear forces, thereby affecting the coagulation and complement cascade ${ }^{7}$. Second, the rotation speed was set to $30 \mathrm{rpm}$ in this experiment. This will result in a blood flow of approximately $25 \mathrm{~cm} / \mathrm{s}$, which is comparable to the blood flow velocity in human coronary artery bypass grafts ${ }^{25}$. The strain rate, generated by the rotation of the loops, is the major parameter that will initiate biochemical cascades of blood components, including cells and cell-free proteins. But as blood is a non-Newtonian fluid, the strain rate will also be influenced by the tube curvature, respectively the length of the tubes that are closed to loops ${ }^{10}$. Whenever the rotation speed or loop size is changed, it is important to consider that the correlation between strain rate and rotation speed is not linear. The correlation between the rotation speed and strain rate is not sufficiently examined until today and further studies are required to investigate these particular parameters ${ }^{10}, 26,27$. However, based on a model for laminar boundary layer, the given tube diameter of $5 \mathrm{~mm}$ and the rotation speed of $25 \mathrm{~cm} / \mathrm{s}$, a rough estimation of the wall shear stress (WSS) would indicate values between 2.20-22.00 pascal for a distance of $1,00-0,01 \mathrm{~mm}$ to the wall of the tube when the blood density is estimated to be $1060 \mathrm{~kg}^{*} \mathrm{~m}^{-3}$ and the kinetical viscosity is set to 0.0025 pascal $^{*} \mathrm{~s}^{28,29}$. Interestingly, also a more detailed computational analysis of flow dynamics in the curvature of human coronary arteries showed WSS values ranging from 11.33 to 16.77 pascal at roughly comparable parameters for the velocity, density and viscosity of the blood ${ }^{30}$.

Beside this limitation, the presented loop model is a pressure less system, that does not mimic the intravascular blood pressure ratios of the human vascular system.

Next important limitation is that the blood is in contact with air inside the loops, which brings additional interferences. Such a blood-air contact is impacted by two parameters, which includes the gas permeability of the tubes and the retainment of air inside the loops while filling them with blood. Every tube material possesses a certain gas permeability that can lead to significant changes in gas concentrations inside the tubes. While some authors state that the resultant effect of the gas permeability on activation of blood components remains unclear ${ }^{31}$, it is known that the function of the blood coagulators is highly sensitive to a $\mathrm{pH}$-shift, that may be caused by $\mathrm{CO}_{2}$ diffusion $32,33,34$. Here, we have tested the biocompatibility of blood perfusion tubes under indoor air conditions, comparable to clinical scenarios of extracorporeal blood perfusion. For future improvements of the presented model, incubation of the whole model in a $\mathrm{CO}_{2}$ incubator and performing blood $\mathrm{pH}$ validation before and after incubation might be useful to further standardize this model.

Also, the blood-air interface inside the loops can lead to activation of plasma proteins and cell fractions of the blood $^{35,36}$. The roller pump driven devices without air inside the tubes may avoid the issue of blood-air interface, but they certainly induce damage to blood cells with significant elevated levels of hemoglobin compared to the here presented loop model, and the hemoglobin in plasma can interfere with the sensitivity of tested analytes in ELISA ${ }^{16}$. 
In this study we have shown that the hemolytic effect of the loop model itself remains minimal while using biocompatible materials such as heparin coated PVC tubes. Thus, the model is, on the one hand, not causing excessive cell damage compared to pump driven models, but on the other hand inducing plasma proteins due to blood air contact. Of note, van Oeveren et al. developed a ball-valve based loop model avoiding air inside the loops ${ }^{16}$. This promising alternative to the here presented loop model may overcome the problem of the blood-air interface, however, compared to the model presented here, platelet adhesion is still higher for the ballvalve based loop model.

With regard to the static control, it is of note that glass itself has been shown to be a potent activator of the coagulatory system $^{37}$. However, in the presented setup, incubation in a glass beaker (static control) did not lead to excessive host cell activation or activation of the coagulatory system compared to the baseline levels directly after drawing the blood. In conclusion, it might be helpful to use for example polypropylene tubes, if the static control shows high levels of activation.

Regardless of whether it is a loop based or a pump-driven model, these in vitro models completely lack the authentic biological interactions that are mainly contributed by an intact endothelium, which is an ideal blood contacting surface. The rationale behind this issue is more evident when a medical device like a stent is being tested, which might impart different outcomes, in terms of activation and plasma proteins, during its interaction with blood components in the presence of endothelium. This declares to be a major drawback of all discussed in vitro systems mimicking the circulatory system. Hence, to overcome this issue, new microfluidic systems that are completely covered with endothelium are gaining immense interest, but nevertheless in comparison to the loop model presented here, they are still limited to accommodate smaller blood volumes and minimal flow rates ${ }^{38}, 39$

Thus, we conclude that the Chandler Loop model remains to be a robust model for conducting standardized tests on the blood biocompatibility of vascular medical devices in the field of cardiovascular research.

\section{Disclosures}

The funder [ebo kunze industriedesign, Im Dentel 17, 72639 Neuffen, Germany] provided a financial support in the form of consumables and publication fees to the author of this manuscript [Max Wacker]. The funders did not have any additional role in the study design, data collection and analysis, decision to publish, or preparation of the manuscript.

\section{Acknowledgments}

The authors are thankful to Ms. Elena Denks for her technical assistance.

\section{References}

1. International Organisation for Standardisation. DIN ISO 10993-4: Biological evaluation of medical devices - Part 4: Selection of tests for interactions with blood. (2017).

2. Mayes, J. T., Schreiber, R. D., Cooper, N. R. Development and application of an enzyme-linked immunosorbent assay for the quantitation of alternative complement pathway activation in human serum. Journal of Clinical Investigation. 73 (1), 160-170 (1984).

3. Maiolini, R. et al. A sandwich method of enzymeimmunoassay. II. Quantification of rheumatoid factor. Journal of Immunological Methods. 20, 25-34 (1978). 
4. Shapiro, H. M. Flow Cytometry: The Glass Is Half Full. Methods in Molecular Biology. 1678, 1-10 (2018).

5. Betke, U. et al. Impact of Slurry Composition on Properties of Cellular Alumina: A Computed Tomographic Study. Advanced Engineering Materials. 19 (10), (2017).

6. Chandler, A. B. In vitro thrombotic coagulation of the blood; a method for producing a thrombus. Laboratory Investigation. 7 (2), 110-114 (1958).

7. Fink, H. et al. An in vitro study of blood compatibility of vascular grafts made of bacterial cellulose in comparison with conventionally-used graft materials. Journal of Biomedical Materials Research Part A. 97 (1), 52-58 (2011).

8. Lenz-Habijan, $T$. et al. Comparison of the Thrombogenicity of a Bare and Antithrombogenic Coated Flow Diverter in an In Vitro Flow Model. Cardiovascular and Interventional Radiology. 43 (1), 140-146 (2020).

9. Olsen, A. L., Long, M. Comparison of catheter thrombogenicity in a modified chandler loop model using goat blood. Journal of Biomedical Materials Research Part A. 106 (12), 3143-3151 (2018).

10. Touma, H., Sahin, I., Gaamangwe, T., Gorbet, M. B., Peterson, S. D. Numerical investigation of fluid flow in a chandler loop. Journal of Biomechanical Engineering. 136 (7), (2014).

11. Slee, J. B., Alferiev, I. S., Levy, R. J., Stachelek, S. J. The use of the ex vivo Chandler Loop Apparatus to assess the biocompatibility of modified polymeric blood conduits. Journal of Visualized Experiments. (90), e51871 (2014).

12. Feyerabend, F. et al. Blood compatibility of magnesium and its alloys. Acta Biomaterialia. 25, 384-394 (2015).
13. Lukas, K. et al. Effect of Immobilized Antithrombin III on the Thromboresistance of Polycarbonate Urethane. Materials (Basel, Switzerland). 10 (4), e355 (2017).

14. Paul, A. et al. Aptamers influence the hemostatic system by activating the intrinsic coagulation pathway in an in vitro Chandler-Loop model. Clinical and Applied Thrombosis/Hemostasis. 16 (2), 161-169 (2010).

15. Link, A. et al. Hemocompatibility Testing of BloodContacting Implants in a Flow Loop Model Mimicking Human Blood Flow. Journal of Visualized Experiments. (157), e60610 (2020).

16. van Oeveren, W., Tielliu, I. F., de Hart, J. Comparison of modified chandler, roller pump, and ball valve circulation models for in vitro testing in high blood flow conditions: application in thrombogenicity testing of different materials for vascular applications. International Journal of Biomaterials. 2012, 673163 (2012).

17. Maa, Y. F., Hsu, C. C. Protein denaturation by combined effect of shear and air-liquid interface. Biotechnology and Bioengineering. 54 (6), 503-512 (1997).

18. Mutch, N. J. et al. The use of the Chandler loop to examine the interaction potential of NXY-059 on the thrombolytic properties of rtPA on human thrombi in vitro. British Journal of Pharmacology. 153 (1), 124-131 (2008).

19. Fletcher, E. A. K. et al. Extracorporeal human whole blood in motion, as a tool to predict first-infusion reactions and mechanism-of-action of immunotherapeutics. International Immunopharmacology. 54, 1-11 (2018).

20. Krajewski, S. et al. Hemocompatibility evaluation of different silver nanoparticle concentrations employing a modified Chandler-loop in vitro assay on human blood. Acta Biomaterialia. 9 (7), 7460-7468 (2013). 
21. Larm, O., Larsson, R., Olsson, P. A new nonthrombogenic surface prepared by selective covalent binding of heparin via a modified reducing terminal residue. Biomaterials, Medical Devices, and Artificial Organs. 11 (2-3), 161-173 (1983).

22. Gong, J. et al. Tubing loops as a model for cardiopulmonary bypass circuits: both the biomaterial and the blood-gas phase interfaces induce complement activation in an in vitro model. Journal of Clinical Immunology. 16 (4), 222-229 (1996).

23. Tevaearai, H. T. et al. Trillium coating of cardiopulmonary bypass circuits improves biocompatibility. The International Journal of Artificial Organs. 22 (9), 629-634 (1999).

24. Ma, Y. Q., Plow, E. F., Geng, J. G. P-selectin binding to P-selectin glycoprotein ligand-1 induces an intermediate state of alphaMbeta2 activation and acts cooperatively with extracellular stimuli to support maximal adhesion of human neutrophils. Blood. 104 (8), 2549-2556 (2004).

25. Bandyk, D. F., Galbraith, T. A., Haasler, G. B., Almassi, G. H. Blood flow velocity of internal mammary artery and saphenous vein grafts to the coronary arteries. Journal of Surgical Research. 44 (4), 342-351 (1988).

26. Gardner, R. A. An examination of the fluid mechanics and thrombus formation time parameters in a Chandler rotating loop system. Journal of Laboratory and Clinical Medicine. 84 (4), 494-508 (1974).

27. Gaamangwe, T., Peterson, S. D., Gorbet, M. B. Investigating the Effect of Blood Sample Volume in the Chandler Loop Model: Theoretical and Experimental Analysis. Cardiovascular Engineering and Technology. 5 (2), 133-144 (2014).
28. Böswirth, L. Technische Strömungslehre. 8 edn. Springer Vieweg. (2010).

29. Cartwright, I. J., Pockley, A. G., Galloway, J. H., Greaves, M., Preston, F. E. The effects of dietary omega-3 polyunsaturated fatty acids on erythrocyte membrane phospholipids, erythrocyte deformability and blood viscosity in healthy volunteers. Atherosclerosis. 55 (3), 267-281 (1985).

30. Wong, K. K. L., Wu, J., Liu, G., Huang, W., Ghista, D. N. Coronary arteries hemodynamics: effect of arterial geometry on hemodynamic parameters causing atherosclerosis. Medical \& biological engineering \& computing. 58, 1831-1843 (2020).

31. Kania, R. E., Herman, P., Ar, A., Tran Ba Huy, P. Technical pitfalls in middle ear gas studies: errors introduced by the gas permeability of tubing and additional dead space. Acta Oto-Laryngologica. 125 (5), 529-533 (2005).

32. Foley, M. E., McNicol, G. P. An in-vitro study of acidosis, platelet function, and perinatal cerebral intraventricular haemorrhage. The Lancet. 1 (8024), 1230-1232 (1977).

33. Engstrom, M., Schott, U., Romner, B., Reinstrup, P. Acidosis impairs the coagulation: A thromboelastographic study. The Journal of Trauma. 61 (3), 624-628 (2006).

34. Dirkmann, D., Hanke, A. A., Gorlinger, K., Peters, J. Hypothermia and acidosis synergistically impair coagulation in human whole blood. Anesthesia \& Analgesia. 106 (6), 1627-1632 (2008).

35. Ritz-Timme, S., Eckelt, N., Schmidtke, E., Thomsen, H. Genesis and diagnostic value of leukocyte and platelet accumulations around "air bubbles" in blood after venous 
air embolism. International Journal of Legal Medicine. 111

(1), 22-26 (1998).

36. Thorsen, T., Klausen, H., Lie, R. T., Holmsen, H. Bubbleinduced aggregation of platelets: effects of gas species, proteins, and decompression. Undersea \& Hyperbaric Medicine : Journal of the Undersea and Hyperbaric Medical Society, Inc. 20 (2), 101-119 (1993).

37. Streller, U., Sperling, C., Hubner, J., Hanke, R., Werner, C. Design and evaluation of novel blood incubation systems for in vitro hemocompatibility assessment of planar solid surfaces. Journal of Biomedical Materials Research Part B. 66 (1), 379-390 (2003).

38. Hesh, C. A., Qiu, Y., Lam, W. A. Vascularized microfluidics and the blood-endothelium interface. Micromachines (Basel). 11 (1), 18 (2019).

39. Nordling, S., Nilsson, B., Magnusson, P. U. A novel in vitro model for studying the interactions between human whole blood and endothelium. Journal of Visualized Experiments. (93), e52112, (2014). 